\title{
Editorial
}

\section{Making Optimal Use of and Extending beyond Polygenic Additive Liability Models}

\author{
John P.A. loannidis \\ Department of Medicine and of Health Research and Policy, Stanford University School of Medicine, and \\ Department of Statistics, Stanford University School of Humanities and Sciences, Stanford, Calif., USA
}

This issue of Human Heredity contains six very interesting articles on polygenic additive liability models and related themes [1-6]. Three papers debate on whether these models are a good approximation to reality. Ott [1] reviews the history of polygenic models starting with the influential early work of R.A. Fisher and shows that a simple polylocus threshold model can work very well for a complex phenotype such as schizophrenia. Visscher and Wray [2] argue further on the success of applying polygenic additive models in diverse complex phenotypes and suggest that these tools can take us quite far in genetic prediction and in understanding genetic architecture. Génin and Clerget-Darpoux [3] conversely take a closer look into the assumptions of polygenic additive liability models and claim that, even in the case of diabetes mellitus (a condition where supposedly this approach has worked well since first proposed over half a century ago [7]), there are still major problems and obvious violations of the key assumptions for these models. Indeed, the major assumptions (that the disease is genetically homogeneous; that dominance and epistasis effects are negligible in the disease etiology, and that neither a genetic nor an environmental factor has a major contribution) may be tenuous for many phenotypes of interest. Génin and Cler-
C 2016 S. Karger AG, Basel

0001-5652/16/0804-0158\$39.50/0
get-Darpoux [3] also make a plea for putting again more research effort and resources towards understanding epistasis and different types of interactions and towards capturing the biological basis of associations, rather than simply expanding the scale of genotyped sample sizes in an effort to identify more and more loci with typically smaller and smaller effects.

The juxtaposition of the views of Visscher and Wray [2] and of Genin and Clerget-Darpoux [3] creates an interesting debate. Visscher and Wray argue with some credible empirical data that epistasis and interactions have been difficult to unearth for many phenotypes to date; this may mean that such large interactions are infrequent. Of course, claims for gene-gene and gene-environment interactions have been extremely frequent in the published literature, but the majority of those presented to date in scattered studies are probably false-positives due to exploratory analyses in an underpowered setting [8]. Visscher and Wray suggest that genuine interactions may still be of high order and this would be difficult to tackle with the current approaches that typically examine only 2 or 3 factors. Regardless, all these interactions may not be so essential to know about for practical purposes (e.g. making an accurate clinical prediction), since em- 
pirical data suggest that polygenic liability threshold models fit the data pretty well for several phenotypes [9$12]$ - as also noted by Ott [1].

A caveat in the arguments of Visscher and Wray is that these models have not been applied in more than a handful of phenotypes, and for most phenotypes of medical interest the amount of data in genetic association studies, consortia and even meta-analyses thereof $[13,14]$ are not yet in the range where one would be able to make conclusive statements about the presence of diverse types of interactions and their magnitude. To offer concrete insights into the potential complexity involved, Genin and Clerget-Darpoux [3] discuss the intricacy of a common phenotype such as diabetes where both genetic and environmental heterogeneity have been clearly demonstrated, and both genetic and environmental large effects have been found, along with many more much smaller effects.

Another three papers in this issue deal with important technical aspects and suggestions on how to improve the accuracy and efficiency of the methods used in polygenetic modeling and how to avoid pitfalls. Dudbridge and Newcombe [4] address a common question when generating multigenic risk scores, i.e. whether pruning should be performed or not for markers in high linkage disequilibrium (LD). They demonstrate that pruning is useful and loses little information in currently available sample sizes, while full consideration of all genetic markers with adjustment for the LD structure may offer more clear advantages when sample sizes become larger, in the millions of participants [4]. Such sample sizes are already feasible and they will probably become more common in the future with the advent of multiple biobanks, as well as widespread genome testing in routine healthcare or even among healthy people, even though the practical value of these behemoth datasets is still unclear. Jiang et al. [5] propose that retrospective association analysis methods (as opposed to the currently widely used prospective association analyses) may help to overcome some of the limitations of the phenotype model, in particular misspecification due to ascertainment. This is an important issue, because, in contrast to genotyping, where error rates and misclassification is currently minimal, problems with ascertainments of phenotypes and phenotype misclassification remain a major challenge for many disciplines. In the era of big data, such mis-specification may actually become even more common and more prominent, since massive collection of data is often accompanied by a decrease in accuracy and fidelity of the information, as in the case of administrative electronic health record datasets. Finally, Dandine-Roulland and Perdry [6] provide an overview of the theory and applications of mixed linear models in human genetics, since these models often provide the statistical backbone of polygenic modeling. They discuss the relevance of modeling genetic effects as random effects and warn against an overinterpretation of the model predictions. They emphasize the fact that if, for rejecting the null hypothesis, a naïve model will usually work, this is not the case when one wants to use this model for prediction purposes [6].

Like any modeling effort, polygenic additive liability models are always likely to deviate from a perfect fit to the data, so the main question is by how much they deviate and whether this deviation matters. The answer to this question is still partly unknown, because despite very promising data, application of these models for real, practical use is still in its infancy. There is a lot of interest in incorporating various genetic scores in clinical practice to improve decision-making and to ameliorate clinical outcomes eventually. A good predictive model should make more than just an accurate prediction. Prediction involves aspects of calibration, discriminating ability, and improved risk reclassification. Furthermore, one needs to demonstrate that the use of this information improves patient outcomes, ideally hard clinical outcomes and not just surrogates. While there is large diversity in the practical performance of such models, it is fair to say that usually known nongenetic risk factors have a much greater contribution towards explaining risk of outcomes than complex genetic scores. Of course, this situation may change in the future, as genetic scores become more informative. After all, a major promise of polygenic models is that they can capture a large share of the genetic risk without necessarily having to pinpoint which specific genetic variants are the most essential ones and the exact magnitude of risk conferred by each of them.

Some other issues need to be acknowledged. The flurry of activity on genetic associations over several decades has generated association data on many thousands of phenotypes. The amount and quality of data varies a lot across phenotypes and accordingly the amount of solidly discovered loci varies substantially from null to many hundreds per phenotype. Phenotype multiplicity may need to be more seriously considered in interpreting results in the future. For example, while many discovered genetic loci have extremely robust statistical support and are replicated repeatedly in independent samples, others have more borderline levels of statistical significance and very heterogeneous results across populations. While earlier evaluations suggested that $\mathrm{p}$ values $<10^{-7}$ detected mostly genuine loci [15], with the rapid expansion of phe- 
notype multiplicity, the threshold for ensuring high credibility may need to become higher. A similar correction may also apply for the performance of polygenic risk scores.

Furthermore, phenotypes are correlated and there can be many variants of the same phenotype definition. These variants may contribute to overall heterogeneity, but they may also cause vibration of effects (different predictive effects depending on how the phenotype is exactly defined) and eventually inflated predictive effects in published estimates of predictive ability, if investigators select the most promising results for publication. Moreover, we need to find ways to account for the lack of stability of many phenotypes over time and acknowledge the fact that the large majority of phenotypes of medical interest are time dependent [16]. The large majority of quantitative traits have prominent changes over time, while even binary phenotypes of disease do not necessarily label individuals for their entire life span, e.g. patients are healthy, then may become diabetic, then may become healthy (normoglycemic) again and then they may relapse into the diabetic range again. Fluctuations in clinical status may be accompanied by marked changes in nongenetic markers [17]. Similar fluctuation would also be seen if one were to use a respective quantitative trait, e.g. hemoglobin $\mathrm{A}_{1 \mathrm{c}}$.

Environmental and lifestyle experiences are clearly influential in shaping the evolution of this time-dependent phenotype experience of individuals. Therefore, the impact of gene-environment interactions may be more interesting to assess not in the typical static case-control studies that contribute the majority of the data to current genetic association analyses, but in longitudinal, lifecourse, long-term follow-up studies where phenotypes change over time and where exposures are captured in massive scale and with sufficient accuracy.

Capturing all this dynamic information is not a simple task. It will require some convergence of population genetics, lifestyle epidemiology, population science and big data approaches. It may also be facilitated by an improved ability to measure the exposurome (or envirome) $[18,19]$ in more depth and with more granularity.

Even defining environmental factors can be a challenge, since these can not only change over time, but their occurrence may also be affected by genetics. For example, smoking behavior, a major determinant of many diseases, is regulated to some extent by genetic factors [20]. Conversely, one may need to revisit whether there can be in fact environmental influence on genetic factors. The classic situation has been when prevalent infectious diseases may eventually select for particular types of genotypes. However, with decreasing impact of infectious diseases and with the advent of exposure- and lifestyle-dependent chronic diseases, could an evolving selection pattern be seen also with noncommunicable diseases? This may appear regardless of whether the genes involved in the interactions confer any change in fertility or probability of generating offspring. For example, with lifestyle-dependent diabetes or obesity increasing very fast in many populations, it would be interesting to see whether the genetic associations of diabetes and obesity remain the same or change over time in magnitude and impact in a population where these modern epidemics unfold. Overall, capturing gene-environment interactions is a challenge and it is likely that our attempts to address it so far have been rather superficial, resulting in multiple claims that are mostly refuted but perhaps also missing some of the most exciting interactions.

\section{References}

$>1$ Ott J: Polygenic models for risk prediction in human genetics. Hum Hered 2015;80:162164.

2 Visscher PM, Wray NR: Concepts and misconceptions about the polygenic additive model applied to disease. Hum Hered 2015; 80:165-170.

-3 Génin E, Clerget-Darpoux F: Revisiting the polygenic additive liability model through the example of diabetes mellitus. Hum Hered 2015;80:171-177.

4 Dudbridge F, Newcombe PJ: Accuracy of gene scores when pruning markers by linkage disequilibrium. Hum Hered 2015;80:178186.
5 Jiang D, Mbatchou J, McPeek MS: Retrospective association analysis of binary traits: overcoming some limitations of the additive polygenic model. Hum Hered 2015;80:187-195.

$\checkmark 6$ Dandine-Roulland C, Perdry H: The use of the linear mixed model in human genetics. Hum Hered 2015;80:196-206

7 Falconer DS: The inheritance of liability to diseases with variable age of onset, with particular reference to diabetes mellitus. Ann Hum Genet 1967;31:1-20.
$>8$ Boffetta P, Winn DM, Ioannidis JP, Thomas DC, Little J, Smith GD, Cogliano VJ, Hecht SS, Seminara D, Vineis P, Khoury MJ: Recommendations and proposed guidelines for assessing the cumulative evidence on joint effects of genes and environments on cancer occurrence in humans. Int J Epidemiol 2012; 41:686-704.

9 Purcell SM, Wray NR, Stone JL, Visscher PM, O’Donovan MC, Sullivan PF, Sklar P: Common polygenic variation contributes to risk of schizophrenia and bipolar disorder. Nature 2009;460:748-752. 
10 Schizophrenia Working Group of the Psychiatric Genomics: Biological insights from 108 schizophrenia-associated genetic loci. Nature 2014;511:421-427.

-11 Moser G, Lee SH, Hayes BJ, Goddard ME, Wray NR, Visscher PM: Simultaneous discovery, estimation and prediction analysis of complex traits using a Bayesian mixture model. PLoS Genet 2015;11:e1004969.

12 Abraham G, Rohmer A, Tye-Din JA, Inouye M: Genomic prediction of celiac disease targeting HLA-positive individuals. Genome Med 2015;7:72.

13 Panagiotou OA, Willer CJ, Hirschhorn JN, Ioannidis JP: The power of meta-analysis in genome-wide association studies. Annu Rev Genomics Hum Genet 2013;14:441-465.
14 Evangelou E, Ioannidis JP: Meta-analysis methods for genome-wide association studies and beyond. Nat Rev Genet 2013;14:379-389.

15 Panagiotou OA, Ioannidis JP; Genome-Wide Significance Project: What should the genome-wide significance threshold be? Empirical replication of borderline genetic associations. Int J Epidemiol 2012;41:273-286.

16 Ioannidis JP, Loy EY, Poulton R, Chia KS: Researching genetic versus nongenetic determinants of disease: a comparison and proposed unification. Sci Transl Med 2009;1:7ps8.

17 Chen R, Mias GI, Li-Pook-Than J, Jiang L, Lam HY, Chen R, Miriami E, Karczewski KJ, Hariharan M, Dewey FE, Cheng Y, Clark MJ, Im H, Habegger L, BalasubramanianS, O'Huallachain M, Dudley JT, Hillenmeyer S, Haraksingh R,
Sharon D, Euskirchen G, Lacroute P, Bettinger K, Boyle AP, Kasowski M, Grubert F, Seki S, Garcia M, Whirl-Carrillo M, Gallardo M, Blasco MA, Greenberg PL, Snyder P, Klein TE, Altman RB, Butte AJ, Ashley EA, Gerstein M, Nadeau KC, Tang H, Snyder M: Personal omics profiling reveals dynamic molecular and medical phenotypes. Cell 2012;148:1293-1307.

18 Rappaport SM, Smith MT: Epidemiology: environment and disease risks. Science 2010. 330:460-461.

19 Patel CJ, Ioannidis JP: Studying the elusive exposome in large scale. JAMA 2014;311:2173-2174.

20 Tobacco and Genetics Consortium: Genomewide meta-analyses identify multiple loci associated with smoking behavior. Nat Genet 2010;42:441-447. 